\title{
Guest Editorial: Recent Advances in Non-destructive Testing Methods
}

\author{
Fabio Tosti ${ }^{1}$ (D) . Amir M. Alani ${ }^{1} \cdot$ Andrea Benedetto $^{2}$ (D) . Andreas Loizos ${ }^{3}$ (D) \\ Francesco Soldovieri ${ }^{4}$ (D)
}

Published online: 1 April 2020

(c) Springer Nature B.V. 2020

A wide range of engineering and geoscience applications today rely on the extensive employment of specialist non-destructive testing (NDT) methods. The stand-alone use of these techniques has become established in many areas given the research progress achieved in the use of electrical, electromagnetic, optical and acoustic NDT methods. The capabilities and potential of these methods have been comprehensively explored and assessed, bringing research up to date. Research efforts have been mostly focussed on achieving new theoretical developments, the advancement of hardware and software components, and on finding new surveying and data processing methods and interpretations. As a result of this, the standard of quality is excellent, and data can be collected very accurately with available technology.

At the same time, the concept of integration between sensing methodologies, in terms of modelling data of different scale domains and resolutions, is becoming a real challenge within the scientific community, and is starting to be recognised as a key research area that could drastically enhance the capabilities of existing NDT technology when faced with new and complex scenarios. This trend is driven by an increase in the demand for more

Fabio Tosti

Fabio.Tosti@uwl.ac.uk

Amir M. Alani

Amir.Alani@uwl.ac.uk

Andrea Benedetto

andrea.benedetto@uniroma3.it

Andreas Loizos

aloizos@central.ntua.gr

Francesco Soldovieri

soldovieri.f@irea.cnr.it

1 School of Computing and Engineering, University of West London (UWL), St Mary's Road, Ealing, London W5 5RF, UK

2 Department of Engineering, Roma Tre University, Via Vito Volterra 62, 00146 Rome, Italy

3 Laboratory of Pavement Engineering, National Technical University of Athens (NTUA), Zografou Campus, 9, Iroon Polytechniou Str., Zografou, 15780 Athens, Greece

4 Institute for Electromagnetic Sensing of the Environment (IREA-CNR), National Research Council, Via Diocleziano 328, 80124 Naples, Italy 
effective solutions for the investigation of non-conventional scenarios. These investigations need to be carried out while maintaining reasonable costs and within feasible time requirements, as multiple methods and equipment and interdisciplinary expertise are involved.

The papers presented in this Special Issue stem from a Session on "Data Fusion, Integration, Correlation and Advances of Non-destructive Testing Methods and Numerical Developments for Engineering and Geosciences Applications" held at the European Geosciences Union (EGU) General Assembly in Vienna, Austria, from 8 to 13 April 2018. The aim of the Session was to provide state-of-the-art contributions of NDT methods and numerical developments, with a special focus on the integration of existing equipment and the development of new algorithms, surveying techniques, methods and prototypes for effective monitoring and assessment. The event was an excellent forum to discuss both new research trends in the application of NDT methods and advances achieved in their complex numerical development. The former discussion incorporated research on the integration of remote sensing and ground-based NDT methods for the comprehensive monitoring and assessment of survey sites at network level, as well as reviews and state-of-the-art applications of ground-penetrating radar (GPR) methods to civil engineering structures and infrastructures. The latter discussion was focussed on modelling and data fusion of multi-scale data sets from different NDT methods and equipment, as well as the latest research findings from stand-alone and combined use of advanced diagnostic methods in areas concerned with conservation and cultural heritage.

In the first section of this Special Issue, Bianchini Ciampoli et al. (2020) provide an overview of the latest research developments in the subject area of non-destructive assessment of transport infrastructures. This research takes as its main focus the integration between satellite remote sensing and ground-based NDT techniques. To elaborate, the potential of using the interferometric synthetic aperture radar (InSAR) technique and highfrequency GPR antenna systems is discussed and a case study with an application to a real-life railway infrastructure is presented. The results demonstrate a clear match between areas of displacement identified by the InSAR technique and areas of decay at the foundation level of the track-bed, detected by using GPR.

The second section of this Special Issue presents reviews and recent applications of GPR methods to civil engineering structures and infrastructures, highlighting the relevance of GPR as a stand-alone geophysical technique for rapid and effective assessment and health monitoring. Plati et al. (2020) provide a comprehensive overview of the geophysical methods available for the assessment of modern roadways. The authors emphasise the importance of integrating GPR and NDT methods based on stress wave theory in order to substantially improve pavement condition assessment (PCA) processes during the service life of roadways. Wang et al. (2020) present a study within the highway engineering area of application, which deals with the real-time estimation of density and thickness of thin asphalt pavement overlay during the compaction stage using GPR. Finite-difference timedomain simulation and a nonlinear optimisation model are used, respectively, to create a numerical model of the pavement, including the dielectric contribution of surface moisture, and to address overlapping and surface moisture issues. Validation field tests demonstrate a high level of accuracy in terms of estimating the asphalt concrete overlay thickness and density. A paper by Artagan et al. (2020) reviews the use of conventional and NDT methods for the assessment and health monitoring of railway infrastructures. The review focuses on state-of-the-art research on stand-alone and the synergetic uses of NDT methods in quality control surveys and routine inspections, for infrastructure maintenance purposes. Tosti and Ferrante (2020) provide an overview of the existing literature on GPR methods for the investigation of reinforced concrete structures. A review of the main destructive 
and NDT methods in the field is presented, and an increasing trend in the use of GPR in reinforced concrete structures is highlighted. Furthermore, a case study is discussed that considers a new methodological and data processing approach for the location of rebars in reinforced concrete structures using a high-frequency dual-polarised antenna system.

The third section of this Special Issue presents research around the subject area of modelling and data fusion of multi-scale data sets from different NDT methods and equipment. Völker et al. (2020) discuss the relevance of applying data fusion to improve the significance of outcomes achieved by the use of NDT methods for the investigation of civil engineering infrastructures. As a result of this, the authors identify a gap in knowledge within the subject area of data fusion as applied to the non-destructive assessment of reinforced concrete structures. A data fusion approach based on logistic training data is applied to a data set of information from half-cell potential mapping, Wenner resistivity, microwave moisture and GPR. The benefit of the presented approach is demonstrated, as compared with the best individual half-cell potential method. Qin et al. (2020) report an overview of electromagnetic (GPR) and seismic methods for the near-surface investigation of geological features. In order to overcome the twofold problem of (1) determining the propagation velocity of the electromagnetic wave from GPR within a layered scenario and (2) the limitations given by the seismic geometry of the Rayleigh wave data to the horizontal resolution and detectability of the properties of the shallow stratum, the authors propose an integrated geophysical system based on GPR and Rayleigh wave information within a data processing and inversion framework. The approach is successfully proven to work for the interpretation of geological information in near-surface surveys. Capozzoli et al. (2020) present the latest beneficial advances in the use of electrical and electromagnetic geophysical methods in civil engineering, with a focus on their application in monitoring subsidence and settlement phenomena. Two case studies of precast buildings are discussed in terms of the joint use of electrical resistivity and GPR techniques to assess structural decay sources. The results demonstrate the viability of merging information from NDT methods collected at different scale domains.

The last section of this Special Issue reports reviews and the latest research findings from the use of stand-alone and combined advanced diagnostic methods in areas concerned with conservation and cultural heritage. Alani and Lantini (2020) present an overview of the existing literature on the subject of the assessment and monitoring of tree roots and their interactions with the soil. A lack of available research-based outputs in the fields of tree root interconnectivity and soil interaction is highlighted within the review provided on the main destructive and NDT methods. To this effect, new research developments are discussed in terms of estimating tree root mass density and health. The last two papers report on the use of NDT methods for cultural heritage applications. Ludeno et al. (2020) discuss the joint use of civil engineering investigation methods and non-invasive electromagnetic sensing technologies for the structural health monitoring of historical and cultural assets. The focus is on the integrated application of linear variable displacement transducers (LVDT) and GPR and their ability to monitor the structural integrity of historical buildings. A case study is presented on the application of these two techniques to the monitoring of cracking at the historic Consoli Palace in Gubbio, Italy. The results demonstrate a consistency between the outputs of both the LVDT and the GPR methods, and the ability of these approaches to monitor the expansion and contraction cycles of cracking in masonry structures due to seasonal temperature variations, and the geometry of the inner walls, respectively. Catapano et al. (2020) report an overview of different electromagnetic imaging techniques, from the microwave to the ultraviolet range of frequencies, for cultural heritage applications. The focus of the review is on the use of ultraviolet fluorescence, infrared 
reflectography, TeraHertz imaging and GPR in this area, and an interesting case study is reported concerning the investigation of an early Renaissance wall painting, The Annunciation by Fra Angelico, integrating the above electromagnetic techniques. The results provide a comprehensive assessment of the painting's structure from the foundation layers of paint all the way to the outermost layers; this could be used to facilitate restoration by identifying areas of potential decay.

To conclude, the research presented in this Special Issue highlights the importance of the integrated use of NDT methods in a wide range of application areas and shows how integrating the capabilities of different NDT techniques allows for the provision of a more robust assessment in geophysical surveys. Within this context, although most of the research is focussed on using information simultaneously from different NDT sources, a new trend is emerging in the use of new theoretical and numerical developments and data-fusion approaches for multi-sensing, multi-scale and multi-resolution diagnostics and monitoring. It can be expected that future research will task itself with further investigations into this particular aspect, such that current achievements in established application areas could be improved, as well as unexplored or more complex scenarios could potentially be investigated. Furthermore, it is worth noting that integration is performed mostly between ground-based NDT methods, where GPR is the most popular technique, with specific applications to conservation and cultural heritage. New research paradigms are emerging in terms of multi-scale approaches: information from satellite remote sensing can be used for the identification of specific areas which can then be prioritised for intervention using ground-based techniques at a later stage. In particular, the wider scale of investigation available from satellite remote sensing is fostering research developments within the context of a more sustainable assessment of transport infrastructures. Further research is needed in this area to develop new data-fusion approaches. In terms of the application of self-standing non-destructive geophysical techniques, GPR is confirmed as the most widespread method for surveying civil engineering structures and infrastructures. Within this context, new research trends appear to focus on niche applications for more comprehensive assessments and the monitoring of structural components.

Acknowledgements The Guest Editors are sincerely grateful to all the referees for their contribution to improving the quality of the research published in this Special Issue. A sincere acknowledgement goes to the Editor-in-Chief of the journal, Professor Michael Rycroft, for the superb supervision and continuous support provided across the whole process as well as to the Springer Nature editorial team for their technical assistance.

\section{References}

Alani AM, Lantini L (2020) Recent advances in tree root mapping and assessment using non-destructive testing methods: a focus on ground penetrating radar. Surv Geophys. https://doi.org/10.1007/s1071 2-019-09548-6

Artagan SS, Bianchini Ciampoli L, D’Amico F, Calvi A, Tosti F (2020) Non-destructive assessment and health monitoring of railway infrastructures. Surv Geophys. https://doi.org/10.1007/s10712-019-09544 $-\mathrm{W}$

Bianchini Ciampoli L, Gagliardi V, Clementini C, Latini D, Del Frate F, Benedetto A (2020) Transport infrastructure monitoring by InSAR and GPR data fusion. Surv Geophys. https://doi.org/10.1007/ s10712-019-09563-7

Capozzoli L, De Martino G, Polemio M, Rizzo E (2020) Geophysical techniques for monitoring settlement phenomena occurring in reinforced concrete buildings. Surv Geophys. https://doi.org/10.1007/s1071 2-019-09554-8 
Catapano I, Ludeno G, Cucci C, Picollo M, Stefani L, Fukunaga K (2020) Noninvasive analytical and diagnostic technologies for studying early renaissance wall paintings. Surv Geophys. https://doi. org/10.1007/s10712-019-09545-9

Ludeno G, Cavalagli N, Ubertini F, Soldovieri F, Catapano I (2020) On the combined use of ground penetrating radar and crack meter sensors for structural monitoring: application to the historical Consoli Palace in Gubbio, Italy. Surv Geophys. https://doi.org/10.1007/s10712-019-09526-y

Plati C, Loizos A, Gkyrtis K (2020) Assessment of modern roadways using non-destructive geophysical surveying techniques. Surv Geophys. https://doi.org/10.1007/s10712-019-09518-y

Qin T, Zhao Y, Hu S, An C, Bi W, Ge S, Capineri L, Bohlen T (2020) An interactive integrated interpretation of GPR and Rayleigh wave data based on the genetic algorithm. Surv Geophys. https://doi. org/10.1007/s10712-019-09543-x

Tosti F, Ferrante C (2020) Using ground penetrating radar methods to investigate reinforced concrete structures. Surv Geophys. https://doi.org/10.1007/s10712-019-09565-5

Völker C, Kruschwitz S, Ebell G (2020) A machine learning-based data fusion approach for improved corrosion testing. Surv Geophys. https://doi.org/10.1007/s10712-019-09558-4

Wang S, Zhao S, Al-Qadi IL (2020) Real-time density and thickness estimation of thin asphalt pavement overlay during compaction using ground penetrating radar data. Surv Geophys. https://doi.org/10.1007/ s10712-019-09556-6

Publisher's Note Springer Nature remains neutral with regard to jurisdictional claims in published maps and institutional affiliations. 